\title{
Determination of the optimal time for tamoxifen treatment in combination with radiotherapy
}

\author{
HYUNSOO JANG ${ }^{1}$, JUNYOUNG BAEK ${ }^{2}$, KYUNG-SOO NAM ${ }^{2}$ and SOYOUNG KIM ${ }^{2}$ \\ Departments of ${ }^{1}$ Radiation Oncology and ${ }^{2}$ Pharmacology, School of Medicine, \\ Dongguk University, Gyeongju, Gyeongsangbuk-do 780-350, Republic of Korea
}

Received June 3, 2016; Accepted August 11, 2016

DOI: $10.3892 /$ ijo.2016.3687

\begin{abstract}
Although radiotherapy and tamoxifen have been extensively used to treat estrogen receptor $\alpha(\mathrm{ER} \alpha)$-positive breast cancers, it is still questionable when tamoxifen should be started to maximize clinical benefits in combination with radiotherapy. Generally, clinician's opinion and experience are major determinants in scheduling concurrent or sequential tamoxifen and radiotherapy. Thus, we attempted to determine an optimal time to start tamoxifen treatment by analyzing tamoxifen responses at different times after irradiating MCF-7 cells to cumulative doses of 10 or 20-30 Gy. MCF-7 cells were irradiated with 5 Gy a week, twice (a cumulative dose of $10 \mathrm{~Gy}$ ) followed by a period of recovery. MTT viability assay for tamoxifen was done with MCF-7 cells harvested immediately after each 5 Gy (MCF-7-5 Gy) or 10 Gy (MCF7-10 Gy) irradiation or after subsequent culture of surviving MCF-7-10 Gy cells for 40 days (MCF-7-R1). To establish the radioresistant cells, the above cycles of irradiation were repeated for a cumulative dose of $20 \mathrm{~Gy}$ (MCF-7-R2) or $30 \mathrm{~Gy}$ (MCF-7-R3). In addition, cytotoxic effects of tamoxifen were also measured. Attenuated tamoxifen response was observed in MCF-7-5 Gy and 10 Gy cells, whereas the efficacy of tamoxifen was restored in MCF-7-R1 cells. Furthermore, these responses to tamoxifen correlated with ER $\alpha$ expression. However, the radioresistant MCF-7 cells (MCF-7-R2/
\end{abstract}

Correspondence to: Dr Soyoung Kim or Dr Kyung-Soo Nam, Department of Pharmacology, School of Medicine, Dongguk University, 87 Dongdae-ro, Gyeongju, Gyeongsangbuk-do 780-350, Republic of Korea

E-mail: soyoungkim@dongguk.ac.kr

E-mail: namks@dongguk.ac.kr

Abbreviations: HER2, human epidermal growth factor receptor 2; ER, estrogen receptor; PR, progesterone receptor; EGFR, epidermal growth factor receptor; PI3K, phosphoinositol-3 kinase; MAPK, mitogen-activated protein kinase; ERK, extracellular signal-regulated kinase; AKT, protein kinase B; GAPDH, glyceraldehyde-3-phosphate dehydrogenase

Key words: radiotherapy, tamoxifen, radioresistance, ER $\alpha$-positive breast cancer
R3) exhibited resistance to tamoxifen without change in ER expression, but the phosphorylation of AKT was increased. Taken together, our data suggest that sequential tamoxifen treatment following radiotherapy is more effective than concurrent treatment. Furthermore, the reduced efficacy of tamoxifen on radioresistant cells indicates that an additional targeted therapy, such as AKT inhibitor treatment, is required to improve tamoxifen response in radioresistant breast cancer.

\section{Introduction}

Surgery, radiotherapy, chemotherapy, endocrine therapy, and molecular targeted therapy are extensively recommended as combinatorial therapies in breast cancer, depending on cancer stage and biomolecular subtype (1). Particularly, radiotherapy is the indispensable treatment modality for loco-regional control after breast conserving therapy and for eradicating cancer cells remaining after surgery (2-4). Besides radiotherapy, endocrine therapy such as aromatase inhibitor or tamoxifen, is another important treatment option since $\sim 70 \%$ of breast cancer patients are positive for estrogen receptor $\alpha$ $(\mathrm{ER} \alpha)(5)$. Generally, endocrine therapy is administered for 3-5 years when breast cancer is ER $\alpha$-positive $(6,7)$. However, despite the fact that combined radiation and endocrine therapy is commonly used nowadays (8-10), it has not been determined when tamoxifen should be started with respect to radiotherapy to maximize clinical benefits. In fact, clinician's opinion and experience are the major determinants of whether concurrent or sequential tamoxifen and radiotherapy are adopted due to a lack of clear guidelines.

Some preclinical studies have shown that pretreatment of breast cancer cells with tamoxifen interferes with the effects of radiotherapy by arresting cells in the G0/G1 phase (11-13). Moreover, concern has been expressed about increased pulmonary and breast fibrosis associated with concurrent tamoxifen and radiotherapy $(14,15)$. Thus, some clinicians may delay tamoxifen therapy until radiotherapy has been completed to avoid possible toxicities. However, others have suggested that pretreatment with tamoxifen enhances the effect of radiation and does not alter the radiosensitivity of breast cancer cells (16). In addition to preclinical studies, several randomized trials have addressed the relative effectivenesses of sequential and concurrent tamoxifen and radiation therapy, but unfortunately, findings were contradictory and no firm conclusions 


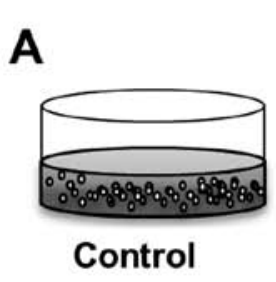

B

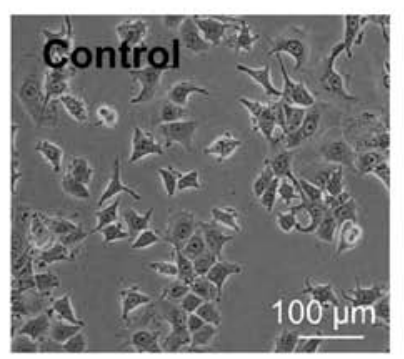

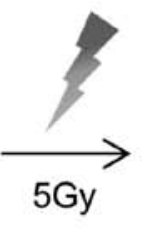

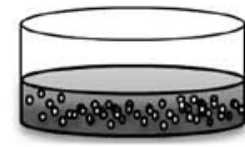

MCF-7-5Gy
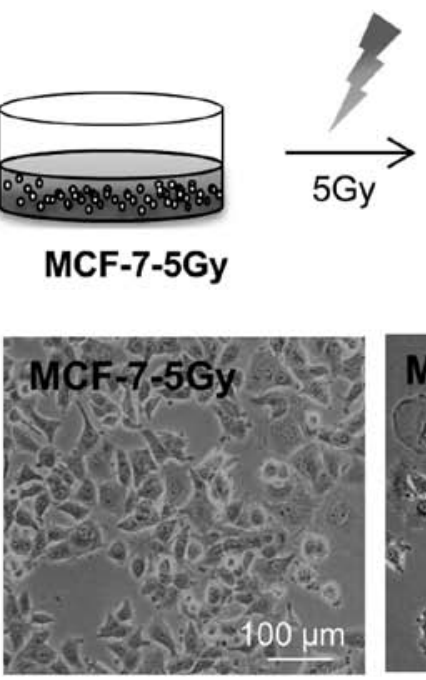
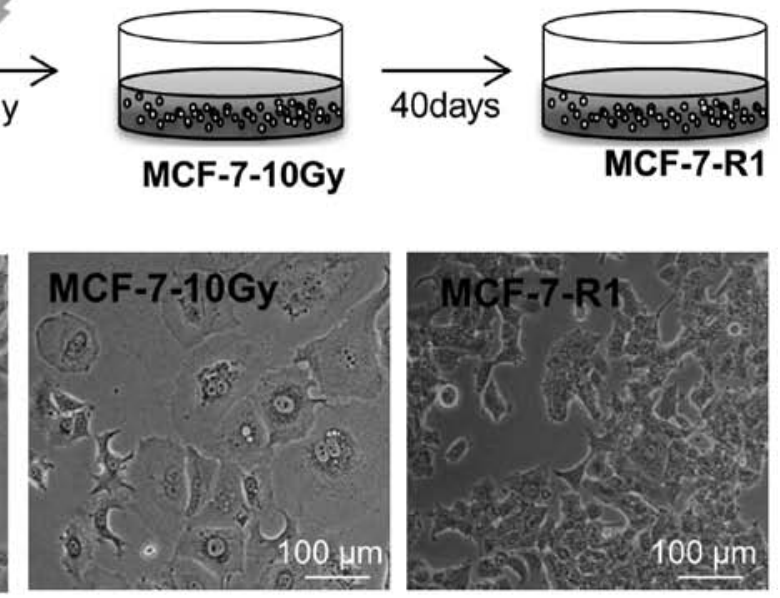

Figure 1. Experimental scheme for measuring the efficacy of concurrent or sequential tamoxifen and radiation treatments. (A) MCF-7 cells were irradiated with 5 Gy (MCF-7-5 Gy), re-cultured for a week and re-irradiated with 5 Gy (MCF-7-10 Gy). A few MCF-7-10 Gy cells survived and slowly recovered over 40 days (MCF-7-R1). (B) Optical microscopic images of irradiated MCF-7 cells at different time-points.

were drawn due, in part, to the small numbers of patients enrolled $(9,17,18)$.

Since the optimal scheduling for tamoxifen and radiotherapy remain unclear, we attempted to identify an optimal time for commencing tamoxifen treatment by analyzing tamoxifen responses in MCF-7 cells at different times after irradiation. In addition, we assessed the effect of tamoxifen in radioresistant cells because tamoxifen has to be administered for several years after radiotherapy and tumors may recur during tamoxifen treatment due to the presence of radioresistant breast cancer cells.

\section{Materials and methods}

Cell culture. MCF-7 cells (a human breast cancer cell line) were purchased from the Korean Cell Line Bank (Seoul, Korea). Cells were cultured in DMEM (WelGENE, Daegu, Korea) supplemented with $10 \%$ fetal bovine serum (Invitrogen Life Technologies, Carlsbad, CA, USA), $1 \%$ antibiotic-antimycotic solution (WelGENE), and $10 \mu \mathrm{g} / \mathrm{ml}$ insulin (WelGENE).

Irradiation and establishment of radioresistant cell lines. MCF-7 cells $\left(1 \times 10^{6}\right)$ were seeded in a $75-\mathrm{cm}^{2}$ culture flask, and irradiated using a 21 EX Linac (Varian Medical Systems, Palo Alto, CA, USA) with 6 MV X-rays at a rate of 3 Gy per minute. The field size was $25 \times 25 \mathrm{~cm}$ and the beam was delivered postero-anteriorly. Cells were irradiated with $5 \mathrm{~Gy}$ and harvested (MCF-7-5 Gy) or maintained for a week, passaged at $80 \%$ confluence, and when in the growth phase irradiated with a second fraction of 5 Gy (MCF-7-10 Gy). MCF-7-5 Gy and MCF-7-10 Gy cells were harvested immediately after each 5 or $10 \mathrm{~Gy}$ irradiation for further experiments. In addition, MCF-7-10 Gy cells were maintained for 40 days to allow them time to recover (MCF-7-R1). The same cycles of irradiation were repeated for a cumulative dose of $20 \mathrm{~Gy}$ (MCF-7-R2) and 30 Gy (MCF-7-R3) over 5 months to establish radioresistant MCF-7 cells.
Colony formation assay. Radioresistance was measured using a clonogenic cell survival assay. MCF-7-R3 and MCF-7 control cells were seeded into 6-well plates at 300-1,200 cells/ well and exposed to 2 or 4 Gy of radiation. All cells were incubated for 10 days at $37^{\circ} \mathrm{C}$ in $5 \% \mathrm{CO}_{2}$; medium was replaced every 3 days. Colonies were fixed with $4 \%$ formaldehyde and stained with $0.01 \%$ crystal violet. Positive colonies, defined as groups of $>50$ cells, were counted manually under a microscope (TS 100, Nikon, Japan). Plating efficiencies of MCF-7-R3 and control cells were determined, and survival fractions were calculated by counting colonies. The experiments were performed in triplicate, and results are presented as means \pm SDs.

Cell viability assay. MCF-7 cells were maintained in phenol-red free DMEM (WelGENE) supplemented with 10\% charcoal-stripped FBS, $1 \%$ antibiotic-antimycotic solution (WelGENE), and $10 \mu \mathrm{g} / \mathrm{ml}$ insulin for 1-2 days prior to the assay. Briefly, 5,000 cells/well were seeded in 96-well plates, incubated at $37^{\circ} \mathrm{C}$ for $24 \mathrm{~h}$, and treated with tamoxifen at 5-20 $\mu \mathrm{M}$ (AG Scientific, San Diego, CA, USA). Cells were then further incubated for $48 \mathrm{~h}$ and viabilities were determined using an MTT assay (Sigma, St. Louis, MO, USA). Absorbance at $570 \mathrm{~nm}$ was measured using a Multi-Detection Microplate Reader (Molecular Devices, Sunnyvale, CA, USA). Experiments were performed in triplicate, and results are presented as means \pm SDs.

Western blot analysis. MCF-7 cells were lysed with RIPA buffer [150 mM NaCl, $1 \%$ Triton X-100, $1 \%$ sodium deoxycholate, $0.1 \%$ SDS, $50 \mathrm{mM}$ Tris- $\mathrm{HCl} \mathrm{pH} 7.5$ and $2 \mathrm{mM}$ EDTA]. Phosphatase and protease inhibitor cocktail (GenDEPOT, Barker, TX, USA) were added to RIPA buffer immediately before use. Total protein concentrations were measured using bicinchoninic acid reagent (Sigma). Proteins were separated in 8 or $10 \%$ SDS-PAGE gels and transferred to polyvinylidene difluoride (PVDF) membranes at $100 \mathrm{~V}$ 
for 45 min, membranes were blocked with $5 \%$ non-fat skim milk containing TBS-Tween $(50 \mathrm{mM}$ Tris- $\mathrm{HCl}, 150 \mathrm{mM}$ $\mathrm{NaCl}, 0.1 \%$ Tween-20) for $1 \mathrm{~h}$ at room temperature. Blots were incubated with the following antibodies at $4^{\circ} \mathrm{C}$ overnight; ER $\alpha$, phosphorylated extracellular signal regulated kinase $1 / 2$ (p-ERK1/2), total-ERK1/2, phosphorylated protein kinase B (p-AKT), total AKT, and $\beta$-actin (Cell Signaling Technology, Beverly, MA, USA). Blots were incubated with horseradish peroxidase-conjugated secondary anti-rabbit antibody (1:5,000; Santa Cruz Biotechnology, Inc., Santa Cruz, CA, USA) for $1 \mathrm{~h}$ at room temperature, and developed using Luminescent Image Analyzer LAS-4000 (Fujifilm, Tokyo, Japan).

$R N A$ isolation and RT-PCR. Total RNA was isolated using the easy-BLUE ${ }^{\mathrm{TM}}$ Total RNA Extraction kit (iNtRON Biotechnology. Inc., Sungnam, Korea) and cDNA synthesis and RT-PCR (reverse transcriptase polymerase chain reaction) were performed as previously described (19). The primer sequences used for the RT-PCR were as follows: ER $\alpha$, forward, 5'-TCC TGATGATTGGTCTCGTCT-3'; reverse, 5'-ACATTTTCCC TGGTTCCTGTC-3'. GAPDH forward, 5'-ATCCCATCACC ATCTTCCAG-3'; and reverse, 5'-TTCTAGACGGCAGGTCA GGT-3'. Densitometric analysis was performed using Scion Image Software (Scion Corp., Frederick, MD, USA).

Flow cytometry. MCF-7 cells were trypsinized and washed with $2 \%$ FBS in phosphate-buffered saline (PBS), incubated with CD24-PE and CD44-FITC (BD Biosciences, San Diego, CA, USA) for $30 \mathrm{~min}$ on ice, washed with $2 \%$ FBS in PBS, and resuspended in a final volume of $500 \mu 1$ PBS buffer for analysis. Fluorescence-activated cell sorting (FACS) was performed using a FACSCalibur II (BD Biosciences). Unstained and single color-labeled samples were used to calibrate the analyzer prior to each experiment.

Statistical analysis. All numerical data are expressed as mean values and standard deviations. The Student's t-test was used to compare mean values. P-values of $<0.05$ were considered statistically significant, and the analysis was performed using SPSS version 18 software.

\section{Results}

Attenuated tamoxifen response in concurrently irradiated MCF-7 cells. As schematically presented in Fig. 1A, MCF-7 cells were irradiated with 5 Gy (MCF-7-5 Gy), re-cultured for a week, and irradiated with a second fraction of $5 \mathrm{~Gy}$ (MCF-7-10 Gy). Interestingly, initial irradiation of cells with 5 Gy (MCF-7-5 Gy) exhibited mild effects on cell viability and a change in cell morphology. However, a second fraction of $5 \mathrm{~Gy}$, resulting in a total dose of $10 \mathrm{~Gy}$ (MCF-7-10 Gy), induced formation of giant cells with aberrant nuclear morphology (Fig. 1B). The formation of giant cells is normally followed by mitotic catastrophe and cell death within a week. However, a few MCF-7-10 Gy cells survived and slowly recovered over 40-50 days (MCF-7-R1 cells). The overall morphology of MCF-7-R1 cells was similar to that of controls (Fig. 1B). Since mitotic catastrophe is the main form of cell
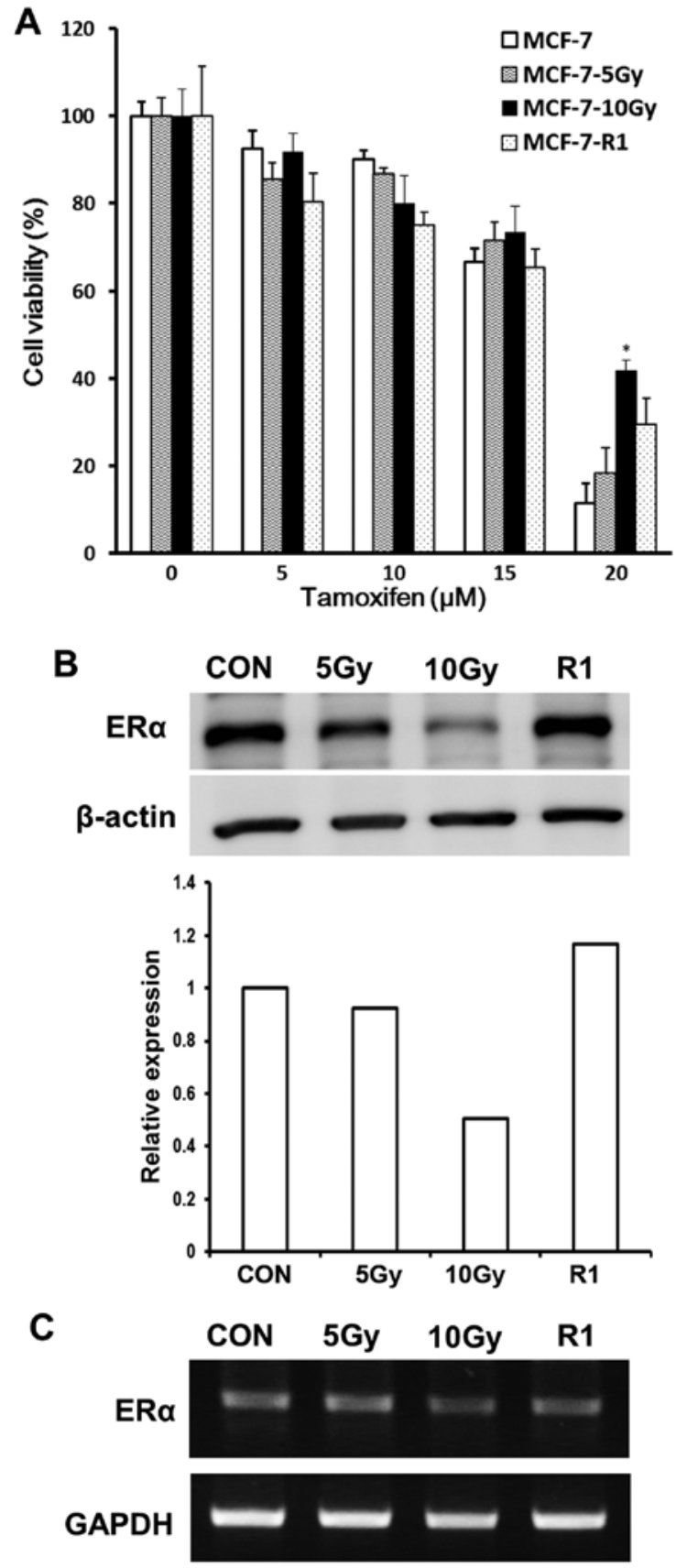

Figure 2. Reduced response to tamoxifen in concurrently irradiated MCF-7 cells was correlated with ER $\alpha$ expression. (A) Cell viability assay to analyze tamoxifen response at different times in MCF-7 cells after irradiation. MTT viability assay was done with MCF-7 cells harvested immediately after each 5 Gy (MCF-7-5 Gy) or 10 Gy (MCF-7-10 Gy) irradiation or after subsequent culture of surviving MCF-10 Gy cells for 40 days (MCF-7-R1). Tamoxifen response diminished in MCF-7-10 Gy cells, but was restored in MCF-7-R1 cells $\left({ }^{*} \mathrm{P}<0.05\right)$. Results are presented as means \pm SDs. The correlation between tamoxifen response and $\mathrm{ER} \alpha$ expression was confirmed by western blotting (B) and RT-PCR (C).

death induced by radiation, we considered MCF-7-5 Gy and MCF-7-10 Gy best represented the clinical situation during radiotherapy, while MCF-7-R1 better represented breast cancer soon after a course of radiation therapy.

To evaluate the efficacy of concurrent and sequential tamoxifen, we tested tamoxifen responses in MCF-7-5 Gy and MCF-7-10 Gy (viewed as representative of concurrent treat- 

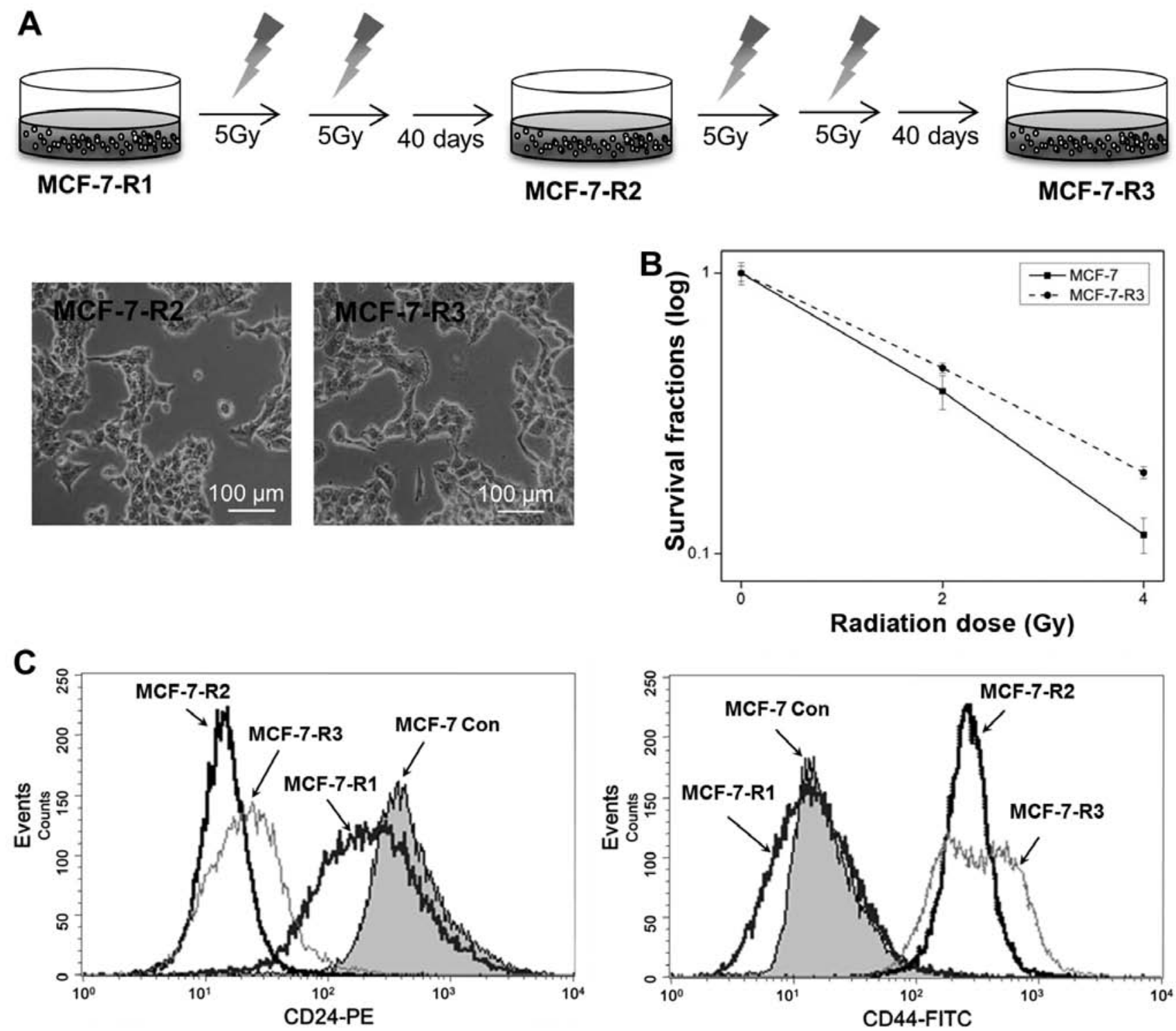

Figure 3. Establishment of radioresistant MCF-7 cells. (A) MCF-7-R1 cells were further cultured and the cycle of irradiation as described in Fig. 1A was repeated to produce MCF-7-R2 and MCF-7-R3 cells. (B) The radioresistance of MCF-7-R3 cells was confirmed by a clonogenic survival assay. (C) Flow cytometry analysis for the expression of CD44 and CD24 in radioresistant MCF-7 cells. The CD44 expression was increased in MCF-7-R2 and MCF-7-R3 cells but the expression of $\mathrm{CD} 24$ was reduced.

ment) or in MCF-7-R1 (sequential treatment) by measuring cell viabilities using the MTT assay. MTT assay was done with MCF-7 cells harvested immediately after each 5 Gy (MCF-7-5 Gy) or 10 Gy (MCF-7-10 Gy) irradiation or after subsequent culture of surviving MCF-7-10 Gy cells for 40 days (MCF-7-R1). Although no significant difference in survival response to tamoxifen was observed between MCF-7-5 Gy and control cells, MCF-7-10 Gy cells exhibited an attenuated response to tamoxifen; at a tamoxifen concentration of $20 \mu \mathrm{M}$ they exhibited $\sim 30 \%$ increase in cell viability versus control cells. However, the efficacy of tamoxifen was restored in MCF-7-R1 cells (Fig. 2A). These observations suggest that sequential tamoxifen treatment is more effective than concurrent treatment.

The correlation between the efficacy of tamoxifen and ERQ expression. Since ER $\alpha$ is the major molecular target of tamox- ifen, we assessed the level of ER $\alpha$ expression in MCF-7-5 Gy, MCF-7-10 Gy, and MCF-7-R1 cells to investigate whether $\mathrm{ER} \alpha$ expression is correlated with the tamoxifen response. Western blot analysis revealed that ER $\alpha$ expression was gradually decreased in MCF-7-5 Gy and remarkably lower in MCF-7-10 Gy cells (Fig. 2B), which also showed the most attenuated response to tamoxifen (Fig. 2A). However, ER $\alpha$ expression and tamoxifen response (Fig. 2A) was recovered in MCF-7-R1 cells (Fig. 2B). Furthermore, RT-PCR analysis showed that the mRNA expression of ER $\alpha$ exhibited a similar pattern observed in western blot analysis (Fig. 2C). Taken together, these results show that response to tamoxifen was positively correlated with ER $\alpha$ expression.

Establishment of radioresistant MCF-7 cells. To establish radioresistant MCF-7 cells, MCF-7-R1 cells were further irradiated as described in Fig. 1A to cumulative doses of 
A
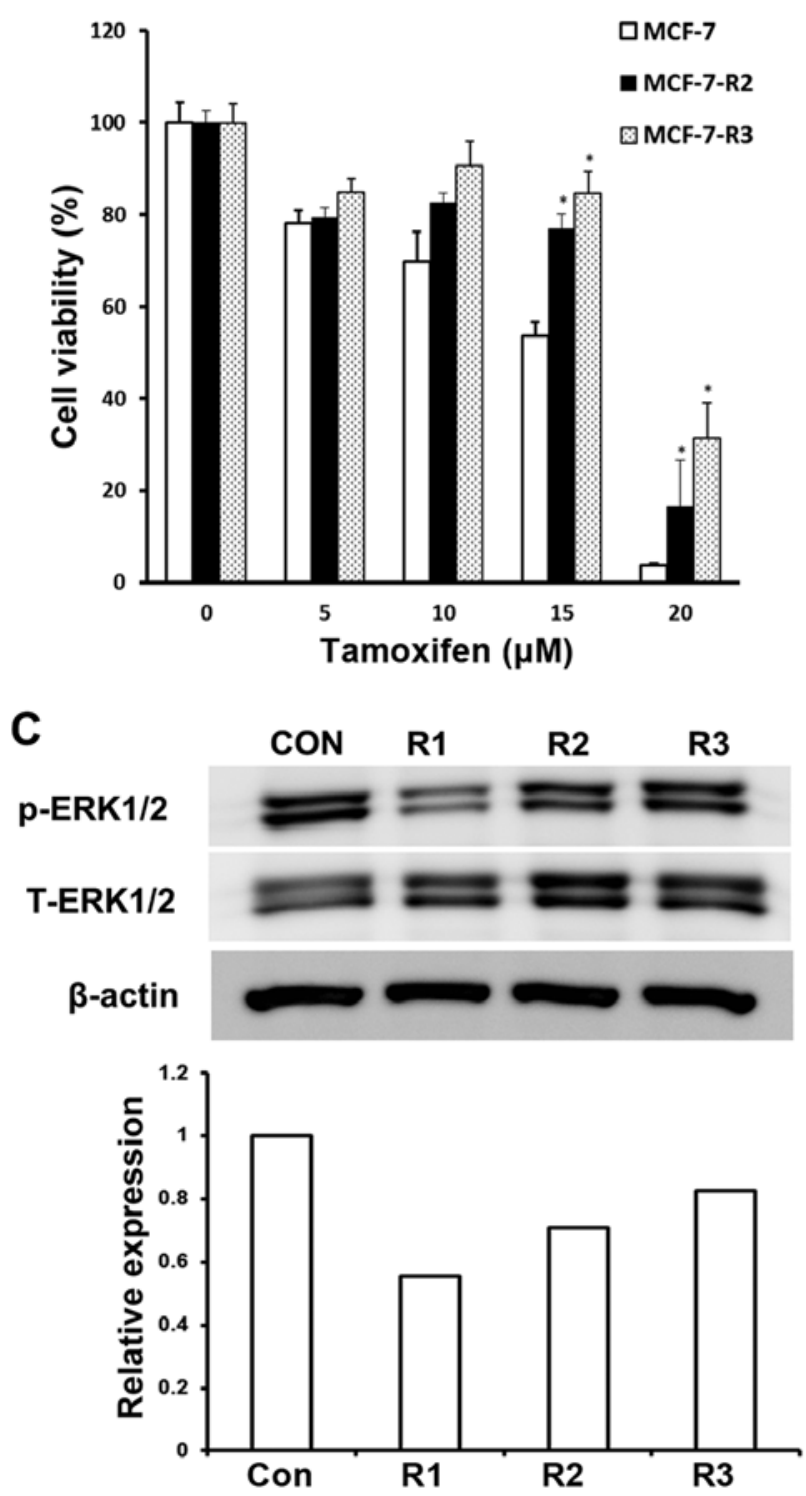

B
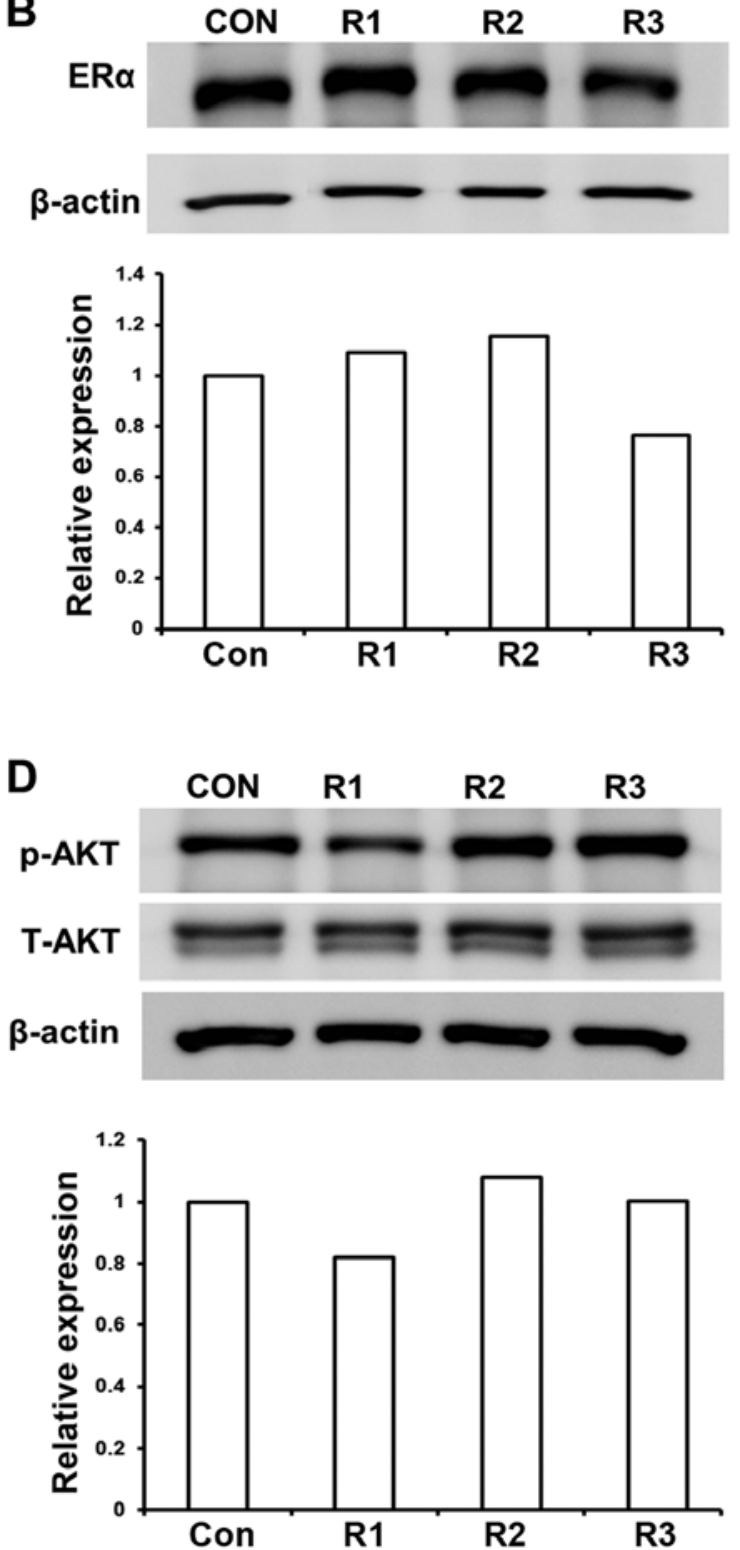

Figure 4. Radioresistant MCF-7 cells exhibited resistance to tamoxifen without change in ER $\alpha$ expression, but the phosphorylation of AKT was increased (A) Response of MCF-7-R2 and MCF-7-R3 cells to tamoxifen. Results are presented as the means \pm SDs of three independent experiments performed in triplicate. Western blot analysis for ER $\alpha$ (B) p-ERK1/2 (C) and p-AKT (D) in MCF-7-R2 and MCF-7-R3 cells. The phosphorylation of AKT was enhanced in MCF-7-R2 and MCF-7-R3 cells, but ER $\alpha$ expression was not changed.

20 and 30 Gy for MCF-7-R2 and MCF-7-R3, respectively (Fig. 3A). Interestingly, the more the process was repeated, the lesser the recovery period was. To confirm the radioresistance of MCF-7-R3, MCF-7 control and MCF-7-R3 cells were irradiated with 2-4 Gy and clonogenic survival assays were performed. As shown in Fig. 3B, MCF-7-R3 exhibited radioresistance; survival fractions at $2 \mathrm{~Gy}$ for control and MCF-7-R3 cells were 0.37 and 0.46 , respectively.

Since cell morphology of MCF-7-R2 and R3 resembled that of cells with an epithelial-mesenchymal transition phenotype, exhibiting fibroblast and mesenchymal characteristics (Fig. 3A), we analyzed the expression of cell-surface proteins, CD44 and CD24, in MCF-7-R2 and R3 cells by flow cytometry. The majority of MCF-7 control cells were of the CD24+/ CD44 phenotype and MCF-7-R1 cells also exhibited this phenotype. However, the expression of CD44 was increased in MCF-7-R2 and R3 cells and CD24 was barely expressed (Fig. 3C), which suggested that cell characteristics were completely changed in radioresistant cells.

Radioresistant MCF-7 cells were also tamoxifen resistant with no change in ER $\alpha$ expression. Generally, tamoxifen treatment is recommended for 3-5 years in ER $\alpha$-positive breast cancer patients after radiotherapy $(6,7)$, and thus, there is a risk of tumor recurrence during the long tamoxifen treatment period. This suggests that the efficacy of tamoxifen in radioresistant cells needs to be assessed to maximize the clinical benefits of tamoxifen in cases that recur after radiotherapy. To evaluate the efficacy of tamoxifen in radioresistant cells, MCF-7-R2 and MCF-7-R3 cells were exposed to different concentrations 
of tamoxifen and cell viabilities were determined. As shown in Fig. 4A, MCF-7-R2 and MCF-7-R3 cells were less sensitive to tamoxifen; they exhibited $>30 \%$ increased viability than the control cells. Furthermore, the resistances of MCF-7-R2 and $-\mathrm{R} 3$ cells to tamoxifen was greater at the higher concentrations tested (Fig. 4A). Since attenuated tamoxifen response in concurrently irradiated MCF-7 cells was found to be positively correlated with ER $\alpha$ expression (Fig. 2), we measured the expression of ER $\alpha$ in MCF-7-R2 and -R3 cells. Interestingly, the expression of $\mathrm{ER} \alpha$ was not altered in these MCF-7-R2 and -R3 cells (Fig. 4B), which implies that the $\mathrm{ER} \alpha$ signaling pathway was not involved in the tamoxifen resistance exhibited by MCF-7-R2 and -R3 cells.

Enhanced AKT activation in radioresistant MCF-7 cells. To understand the mechanism underlying cellular resistance to tamoxifen, we investigated signaling pathways in MCF-7-R2 and $-\mathrm{R} 3$ cells. We first evaluated molecules associated with the non-genomic ER $\alpha$ signaling pathway including EGFR, HER2, mitogen-activated protein kinase/extracellular-signalregulated kinases (MAPK/ERK), and AKT (20-23). No changes in phosphorylated HER2 and phosphorylated EGFR levels were observed in MCF-7-R2 and -R3 cells (data not shown). Phosphorylated ERK1/2 levels were depressed in MCF-7-R1 cells but recovered in MCF-7-R2 and MCF-7-R3 cells (Fig. 4C), suggesting that phosphorylated ERK1/2 is associated with response to tamoxifen in concurrently irradiated MCF-7-R1 cells, but not in MCF-7-R2 and MCF-7-R3 cells. On the other hand, phosphorylated AKT levels were enhanced in MCF-7-R2 and MCF-7-R3 cells versus control cells (Fig. 4D), implying that constitutive AKT activation in radioresistant cells promotes resistance to tamoxifen.

\section{Discussion}

In this study, we tried to identify the optimal time to start tamoxifen treatment in ER $\alpha$-positive breast cancer patients to maximize clinical benefits in combination with radiotherapy. Some previous studies have evaluated the relative effectiveness of sequential and concurrent tamoxifen treatment in this context, but the results obtained were inconsistent $(11-13,16)$. Furthermore, the majority of preclinical studies did not consider the timing of tamoxifen administration relative to radiotherapy, and only analyzed the radiosensitivity of breast cancer after tamoxifen treatment. However, we considered the timing and sequencing of tamoxifen should be applied to reflect the clinical situation. Thus, in this study, we classified irradiated MCF-7 cells according to the course of clinical treatment by treating them with tamoxifen during, immediately and several months after radiotherapy.

In clinical practice, breast radiation is most commonly given 5 days a week for $\sim 5$ or 6 weeks. During the course of radiotherapy, DNA is damaged in tumor cells and this triggers mitotic catastrophe, which is considered to be the major mechanism of cell death induced by radiation in solid tumors $(24,25)$. It is known that mitotic death is caused by aberrant mitosis and subsequent giant cell formation $(26,27)$. In this study, we observed the formation of giant cells when MCF-7 cells were irradiated with a second dose of $5 \mathrm{~Gy}$ (MCF7-10 Gy). However, after the formation of giant cells followed by mitotic catastrophe, a few cells survived and returned to the normal cell cycle (MCF-7-R1). Thus, we considered that MCF-7-5 Gy and MCF-7-10 Gy cells represented the clinical situation during radiotherapy, and MCF-7-R1 cells, which survived mitotic catastrophe, represented breast cancer cells soon after radiotherapy. When we evaluated the efficacy of tamoxifen at different times after irradiation, attenuated tamoxifen response was observed in MCF-7-10 Gy cells, but not in MCF-7-R1 cells, which suggests that tamoxifen is ineffective when aberrant mitosis had occurred by radiation. Furthermore, we found the expression of ER $\alpha$ was diminished when giant cells were formed, presuming that radiationinduced aberrant mitosis reduces ER $\alpha$ expression, resulting in the decreased response to tamoxifen. Taken together, our data suggested that sequential tamoxifen treatment following radiotherapy would be optimal instead of the concurrent treatment.

As mentioned above, patients generally receive radiotherapy for 5-6 weeks, whereas tamoxifen is recommended for 3-5 years in ER $\alpha$-positive breast cancer patients after radiotherapy $(6,7)$. To investigate the efficacy of tamoxifen on recurred tumors due to surviving radioresistant cells, we established the radioresistant MCF-7 cell lines, MCF-7-R2 and MCF-7-R3. Interestingly, MCF-7-R3 cells exhibited resistance to tamoxifen without exhibiting aberrant mitosis or reduction in ER $\alpha$ expression. Although the expression of ER $\alpha$ was not altered in radioresistant MCF-7-R2 and MCF-7R3, they were distinguishable from parental MCF-7 cells or MCF-7-R1 by the upregulation of CD44 and downregulation of CD24. CD44 and CD24 are cell surface glycoproteins that participate in cell-matrix and cell-cell interactions $(28,29)$. Furthermore, a subset of $\mathrm{CD} 24 \% \mathrm{CD} 44^{+}$cells were found to be cancer stem cells in human breast cancer (30). We observed that normal control and MCF-7-R1 cells were of the CD24 ${ }^{+} / \mathrm{CD} 444^{-}$subtype, whereas radioresistant MCF-7-R2 and MCF-7-R3 cells exhibited the CD24/CD44+ subtype even after 3 months, implying that the changes of cell characteristics may contribute to the resistance to both radiation and tamoxifen treatment.

Several studies have shown that PI3K/AKT, HER2, and MAPK/ERK signaling are associated with radioresistance (31-36). Ahmed et al reported that total ERK1/2 is slightly increased and phosphorylated ERK1/2 is decreased in radioresistant MCF-7 cells (31). Chang et al found that the PI3K/ AKT/mTOR signaling pathway is activated in radioresistant prostate cancer cells (36). In this study, we evaluated the expression of AKT, ERK1/2, HER2, and EGFR in radioresistant cells, and found that phosphorylated AKT was increased in MCF-7-R2 and MCF-7-R3 cells, but phosphorylated HER2 and EGFR were not (data not shown). Since activation of the PI3K-AKT signaling pathway is associated with the radioresistance of many cancers by increasing the rate of DNA repair (37), and AKT is related to the non-genomic ER $\alpha$ pathway (38), our data suggest that constitutive AKT activation may contribute to the resistance shown by MCF-7-R2 and -R3 cells to radiation and tamoxifen.

The main goal of this study was to propose an optimal schedule for tamoxifen and radiotherapy. Obviously, extensive clinical studies on a large number of patients are the best way to address this issue, but this clinical approach is demanding 
in terms of time and money. Although this study was conducted in vitro using breast cancer cells, our experimental scheme considered the timing and sequencing of tamoxifen administration so as to reflect the clinical situation. Based on the tamoxifen response and the status of ER $\alpha$ expression shown by irradiated and non-irradiated MCF-7 cells, our findings propose that tamoxifen treatment after radiotherapy is a better treatment option than concurrent treatment. However, the observed reduced efficacy of tamoxifen on radioresistant cells, which showed normal ER $\alpha$ expression, suggests that an additional targeted therapy, such as, AKT inhibitor therapy, is required to improve radioresistant breast cancer response to tamoxifen.

\section{Acknowledgements}

This study was supported by a grant from the Basic Science Research Program, the National Research Foundation of Korea (NRF), funded by the Ministry of Education, Science and Technology, Republic of Korea (grant no. NRF-2015R1D1A1A01059738).

\section{References}

1. National Comprehensive Cancer Network Clinical Practice Guidelines in Oncology: 2014. http://www.nccn.org.

2. Komoike Y, Akiyama F, Iino Y, Ikeda T, Akashi-Tanaka S, Ohsumi S, Kusama M, Sano M, Shin E, Suemasu K, et al: Ipsilateral breast tumor recurrence (IBTR) after breastconserving treatment for early breast cancer: Risk factors and impact on distant metastases. Cancer 106: 35-41, 2006.

3. Arriagada R, Lê MG, Contesso G, Guinebretière JM, Rochard F and Spielmann M: Predictive factors for local recurrence in 2006 patients with surgically resected small breast cancer. Ann Oncol 13: 1404-1413, 2002.

4. Ha B, Suh HS, Lee J, Lee KJ, Lee R and Moon BI: Long-term results of forward intensity-modulated radiation therapy for patients with early-stage breast cancer. Radiat Oncol J 31: 191-198, 2013.

5. Masood S: Estrogen and progesterone receptors in cytology: A comprehensive review. Diagn Cytopathol 8: 475-491, 1992.

6. Early Breast Cancer Trialists' Collaborative Group (EBCTCG): Effects of chemotherapy and hormonal therapy for early breast cancer on recurrence and 15-year survival: An overview of the randomised trials. Lancet 365: 1687-1717, 2005.

7. Fisher B, Dignam J, Bryant J and Wolmark N: Five versus more than five years of tamoxifen for lymph node-negative breast cancer: Updated findings from the National Surgical Adjuvant Breast and Bowel Project B-14 randomized trial. J Natl Cancer Inst 93: 684-690, 2001.

8. Azria D, Pelegrin A, Dubois JB, Mirimanoff RO and Ozsahin M: Radiation therapy and tamoxifen: concurrent or sequential? It's no longer the question! J Clin Oncol 23: 4239-4241; author reply 4241-4232, 2005.

9. Harris EE, Christensen VJ, Hwang WT, Fox K and Solin LJ: Impact of concurrent versus sequential tamoxifen with radiation therapy in early-stage breast cancer patients undergoing breast conservation treatment. J Clin Oncol 23: 11-16, 2005.

10. Whelan $\mathrm{T}$ and Levine M: Radiation therapy and tamoxifen: Concurrent or sequential? That is the question. J Clin Oncol 23: $1-4,2005$.

11. Osborne CK, Boldt DH, Clark GM and Trent JM: Effects of tamoxifen on human breast cancer cell cycle kinetics: Accumulation of cells in early G1 phase. Cancer Res 43: 3583-3585, 1983

12. Paulsen GH, Strickert T, Marthinsen AB and Lundgren S: Changes in radiation sensitivity and steroid receptor content induced by hormonal agents and ionizing radiation in breast cancer cells in vitro. Acta Oncol 35: 1011-1019, 1996.

13. Wazer DE, Tercilla OF, Lin PS and Schmidt-Ullrich R: Modulation in the radiosensitivity of MCF-7 human breast carcinoma cells by 17B-estradiol and tamoxifen. Br J Radiol 62: 1079-1083, 1989 .
14. Bentzen SM, Skoczylas JZ, Overgaard M and Overgaard J: Radiotherapy-related lung fibrosis enhanced by tamoxifen. J Natl Cancer Inst 88: 918-922, 1996.

15. Koc M, Polat P and Suma S: Effects of tamoxifen on pulmonary fibrosis after cobalt-60 radiotherapy in breast cancer patients. Radiother Oncol 64: 171-175, 2002.

16. Ellis PA, Saccani-Jotti G, Clarke R, Johnston SR, Anderson E, Howell A, A'Hern R, Salter J, Detre S, Nicholson R, et al: Induction of apoptosis by tamoxifen and ICI 182780 in primary breast cancer. Int J Cancer 72: 608-613, 1997.

17. Ahn PH, Vu HT, Lannin D, Obedian E, DiGiovanna MP, Burtness B and Haffty BG: Sequence of radiotherapy with tamoxifen in conservatively managed breast cancer does not affect local relapse rates. J Clin Oncol 23: 17-23, 2005.

18. Pierce LJ, Hutchins LF, Green SR, Lew DL, Gralow JR, Livingston RB, Osborne CK and Albain KS: Sequencing of tamoxifen and radiotherapy after breast-conserving surgery in early-stage breast cancer. J Clin Oncol 23: 24-29, 2005.

19. Chun SY, Kwon YS, Nam KS and Kim S: Lapatinib enhances the cytotoxic effects of doxorubicin in MCF-7 tumorspheres by inhibiting the drug efflux function of $\mathrm{ABC}$ transporters. Biomed Pharmacother 72: 37-43, 2015.

20. Shou J, Massarweh S, Osborne CK, Wakeling AE, Ali S, Weiss $\mathrm{H}$ and Schiff R: Mechanisms of tamoxifen resistance: Increased estrogen receptor-HER2/neu cross-talk in ER/HER2positive breast cancer. J Natl Cancer Inst 96: 926-935, 2004.

21. Haynes MP, Sinha D, Russell KS, Collinge M, Fulton D, Morales-Ruiz M, Sessa WC and Bender JR: Membrane estrogen receptor engagement activates endothelial nitric oxide synthase via the PI3-kinase-Akt pathway in human endothelial cells. Circ Res 87: 677-682, 2000.

22. Santen RJ, Song RX, McPherson R, Kumar R, Adam L, Jeng MH and Yue W: The role of mitogen-activated protein (MAP) kinase in breast cancer. J Steroid Biochem Mol Biol 80: 239-256, 2002.

23. Kato S, Endoh $\mathrm{H}$, Masuhiro $\mathrm{Y}$, Kitamoto $\mathrm{T}$, Uchiyama $\mathrm{S}$, Sasaki H, Masushige S, Gotoh Y, Nishida E, Kawashima H, et al: Activation of the estrogen receptor through phosphorylation by mitogen-activated protein kinase. Science 270: 1491-1494, 1995.

24. Rupnow BA and Knox SJ: The role of radiation-induced apoptosis as a determinant of tumor responses to radiation therapy. Apoptosis 4: 115-143, 1999.

25. Verheij M: Clinical biomarkers and imaging for radiotherapyinduced cell death. Cancer Metastasis Rev 27: 471-480, 2008.

26. Galluzzi L, Maiuri MC, Vitale I, Zischka H, Castedo M, Zitvogel L and Kroemer G: Cell death modalities: Classification and pathophysiological implications. Cell Death Differ 14: 1237-1243, 2007.

27. Eriksson D, Löfroth PO, Johansson L, Riklund KA and Stigbrand T: Cell cycle disturbances and mitotic catastrophes in HeLa Hep2 cells following 2.5 to $10 \mathrm{~Gy}$ of ionizing radiation. Clin Cancer Res 13: S5501-S5508, 2007.

28. Kristiansen G, Winzer KJ, Mayordomo E, Bellach J, Schlüns K, Denkert C, Dahl E, Pilarsky C, Altevogt P, Guski H, et al: $\mathrm{CD} 24$ expression is a new prognostic marker in breast cancer. Clin Cancer Res 9: 4906-4913, 2003.

29. Lee HJ, Choe G, Jheon S, Sung SW, Lee CT and Chung JH: $\mathrm{CD} 24$, a novel cancer biomarker, predicting disease-free survival of non-small cell lung carcinomas: A retrospective study of prognostic factor analysis from the viewpoint of forthcoming (seventh) new TNM classification. J Thorac Oncol 5: 649-657, 2010.

30. Al-Hajj M, Wicha MS, Benito-Hernandez A, Morrison SJ and Clarke MF: Prospective identification of tumorigenic breast cancer cells. Proc Natl Acad Sci USA 100: 3983-3988, 2003.

31. Ahmed KM, Dong S, Fan M and Li JJ: Nuclear factor-kappaB p65 inhibits mitogen-activated protein kinase signaling pathway in radioresistant breast cancer cells. Mol Cancer Res 4: 945-955, 2006.

32. Kunigal S, Lakka SS, Joseph P, Estes N and Rao JS: Matrix metalloproteinase-9 inhibition down-regulates radiationinduced nuclear factor-kappa B activity leading to apoptosis in breast tumors. Clin Cancer Res 14: 3617-3626, 2008.

33. Duru N, Fan M, Candas D, Menaa C, Liu HC, Nantajit D, Wen Y, Xiao K, Eldridge A, Chromy BA, et al: HER2-associated radioresistance of breast cancer stem cells isolated from HER2negative breast cancer cells. Clin Cancer Res 18: 6634-6647, 2012.

34. No M, Choi EJ and Kim IA: Targeting HER2 signaling pathway for radiosensitization: Alternative strategy for therapeutic resistance. Cancer Biol Ther 8: 2351-2361, 2009. 
35. Lin F, Luo J, Gao W, Wu J, Shao Z, Wang Z, Meng J, Ou Z and Yang G: COX-2 promotes breast cancer cell radioresistance via p38/MAPK-mediated cellular anti-apoptosis and invasiveness. Tumour Biol 34: 2817-2826, 2013.

36. Chang L, Graham PH, Hao J, Ni J, Bucci J, Cozzi PJ, Kearsley JH and $\mathrm{Li} \mathrm{Y}$ : Acquisition of epithelial-mesenchymal transition and cancer stem cell phenotypes is associated with activation of the $\mathrm{PI} 3 \mathrm{~K} / \mathrm{Akt} / \mathrm{mTOR}$ pathway in prostate cancer radioresistance. Cell Death Dis 4: e875, 2013.
37. Clark AS, West K, Streicher S and Dennis PA: Constitutive and inducible Akt activity promotes resistance to chemotherapy, trastuzumab, or tamoxifen in breast cancer cells. Mol Cancer Ther 1: 707-717, 2002 .

38. Guo RX, Wei LH, Tu Z, Sun PM, Wang JL, Zhao D, Li XP and Tang JM: 17 beta-estradiol activates PI3K/Akt signaling pathway by estrogen receptor (ER)-dependent and ER-independent mechanisms in endometrial cancer cells. J Steroid Biochem Mol Biol 99: 9-18, 2006. 